\title{
Advancements in Plasma-Based Decontamination Equipment and Related Metrology
}

\author{
CA Moore $^{1 *}$ and GJ Collins ${ }^{2}$ \\ 1. Vacuum Sciences, Dana Point, CA, USA. \\ 2. Colorado State University, Electrical and Computer Engineering, Fort Collins, CO, USA. \\ * Corresponding author: cam@vacsci.com
}

The use of flowing plasmas for in situ removal of hydrocarbon contamination in electron microscopes is an established practice, typically in a gas media containing oxygen. The decontamination mechanism is understood as excitation of oxygen molecules to the lowest excited molecular state (singlet delta: ${ }^{1} \Delta_{g}$ or $\mathrm{SDO}, \approx 1 \mathrm{eV}$ above the ground state) followed by reactions with surface-adsorbed hydrocarbons, resulting in volatile products. In the absence of de-exciting collisions SDO has a lifetime $>1$ hour thus is well suited for delivery to surfaces 10's of cm away in a vacuum chamber.

However, despite known mechanisms and a wealth of plasma science, available equipment for performing plasma decontamination is primitive on a plasma technology basis. These commercial systems are little-changed from the first systems (undergoing only small evolutionary improvements) and are mutually almost indistinguishable, all consisting of a plasma head assembly connected via a cable bundle to a large electronics enclosure, with computer control using outdated RS-232 communications. There exists a need for both modernization and optimization of the equipment used for vacuum chamber decontamination.

Criteria for an updated and high performance plasma decontamination system can be divided into interconnected areas of improved system design, broadened operational space, and optimized plasma excitation. Overall system design benefits from a fully integrated realization where only power and communications cables (USB and/or Ethernet) need connection, rendering concerns about RF cable lengths irrelevant. Operationally the plasma should operate reliably at pressures from $\approx 10 \mathrm{mTorr}$ to $\approx 100$ mTorr in the plasma volume to maximize SDO generation and transport. Simultaneously a system should operate at powers from $<\approx 2 \mathrm{~W}$ to $>\approx 40 \mathrm{~W}$ to control downstream heat deposition; for a fixed plasma geometry SDO generation becomes sub-linear as higher discharge powers increasingly appear as heat. Plasma excitation can be improved in myriad ways (many of which are proprietary) but some guidelines include: minimize plasma interaction with metallic surfaces in the plasma generation volume (which de-excite SDO and other plasma species), avoid metallic electrodes in vacuum as they often require conditioning which affects reliability, avoid use of 'valve' metals (Al, Ti) as their thin native oxides promote particulate generation [1], and use of an open-bore design as limiting apertures between the plasma and the chamber quench SDO molecules and cause unwanted local heating in the instrument.

To provide a high performance solution for plasma decontamination, Vacuum Sciences has developed and will be releasing the PM-100 plasma system. While the specifications of this product can be found elsewhere, it is useful to point out the notable engineering achievements in this system. Foremost the system is fully integrated into a single enclosure $100 \mathrm{~mm} \times 100 \mathrm{~mm}$ x $200 \mathrm{~mm}$ in size that minimizes both footprint and cabling clutter. Importantly this reduction in hardware components corresponds to a $>50 \%$ reduction in costs (and thus price). Key to this high level of integration was development of a RF generator of adequate power with sufficient efficiency to minimize waste heat. The resulting design is 
capable of RF power over $80 \mathrm{~W}$ with efficiencies $>90 \%$ (although our measurements indicate little advantage to powers over $45 \mathrm{~W}$ ). Another important feature is that the plasma region is confined to a 25 mm quartz tube that extends to the output side at the KF mating flange, ensuring that the plasma touches minimal stainless steel surfaces. We estimate this aspect is crucial to the enhanced plasma generation and efficiency thereof that is observed, as evidenced by the ability to reliably ignite plasmas at $\approx 20$ mTorr with as little as $1 \mathrm{~W}$ of RF power. Figure 1 shows a photograph of this plasma in operation.

Concurrent efforts are directed at developing a method to better assess SDO generation rate. Prior methods [2] relied on measuring mass loss from a deposited hydrocarbon-based material and suffer from inconsistent and unknown layer properties, time-consuming data collection, and the fact that this proxy does not accurately represent the hydrocarbons common in electron microscopes. Instead it is better to directly measure the optical emission at $1.27 \mu \mathrm{m}$ from decaying SDO molecules, as this will eventually allow both parametric optimizations in real-time and direct comparison between systems. However this measurement is demanding since the SDO emission is weak because the oxygen ground state is a triplet state and thus forbidden in the strict sense. To make this measurement we constructed a detector system consisting of a photodiode with an optical filter of band-pass width $200 \mathrm{~nm}$ containing the SDO wavelength, feeding this signal to a low-noise operational amplifier capable of a gain of $1-10 \times 10^{6}$. Results using this detection system will be presented in future publications.

\section{References:}

[1] C Steinbruchel in "Plasma Sources for Thin Film Deposition and Etching", ed. M. Francombe and J. Vossen, (Academic Press, New York).

[2] C Moore et al., International Workshop on EUVL (2013).

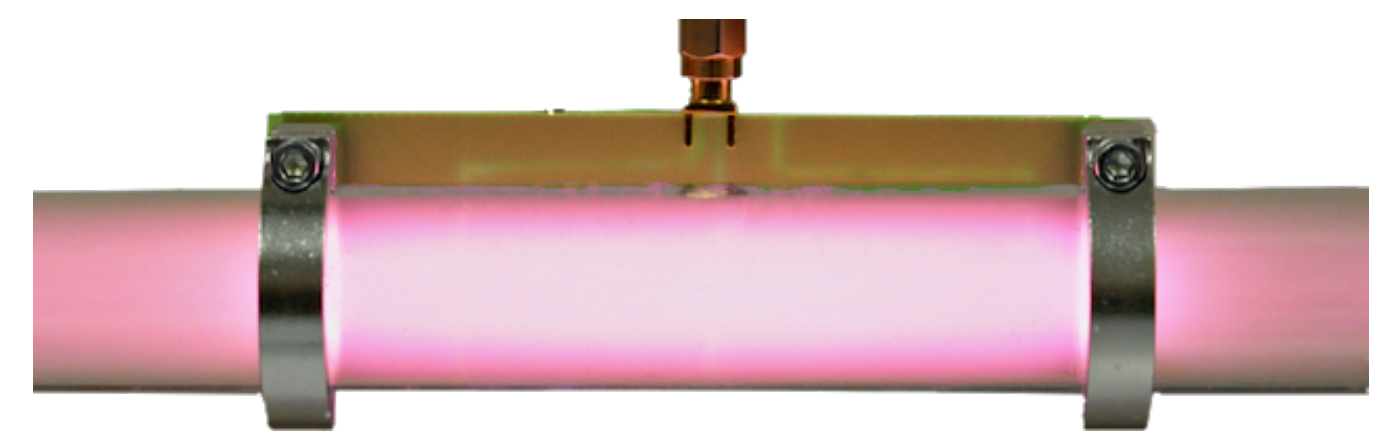

Figure 1. Photograph of high-efficiency plasma system in operation.

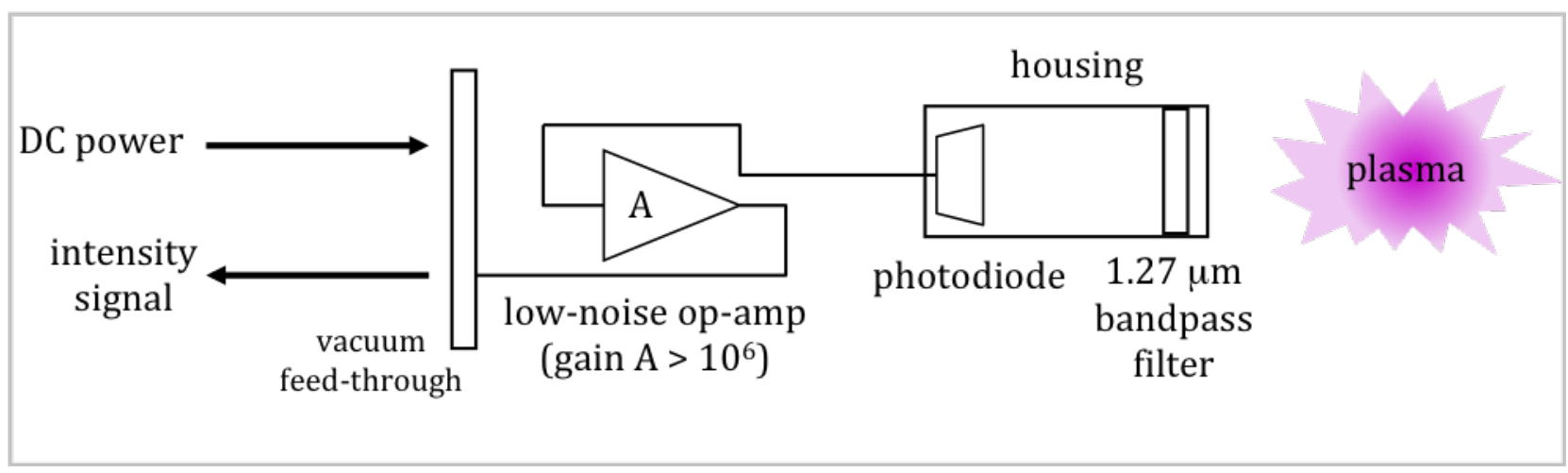

Figure 2. Diagram of system for direct measurement of optical emission from singlet delta oxygen. 